\title{
Radial extracorporeal shock wave therapy for Dupuytren's contracture: a case report
}

\author{
Parisa Taheri $^{*{ }^{(D)}}$, Najmeh Salek ${ }^{\circledR}$, Shervin Ghaffari Hoseini ${ }^{1,2}{ }^{\circledR}$ \\ 'Department of Physical Medicine and Rehabilitation, Isfahan University of Medical Sciences, Alzahra Hospital, Isfahan, Iran \\ ${ }^{2}$ Cardiac Rehabilitation Research Center, Cardiovascular Research Institute, Isfahan University of Medical Sciences, Isfahan, \\ Iran
}

*Corresponding Author: Parisa Taheri, Department of Physical Medicine and Rehabilitation, Isfahan University of Medical Sciences, Alzahra Hospital, Soffe Avenue, Isfahan, Iran.

Postal code: 8174675731, Tel: +983136700666, Mobile: +989131013581,Email: prs_taheri@yahoo.com

\begin{abstract}
Dupuytren's contracture (DC) is a fibro-proliferative disorder of palmar and digital fascia, which leads to contracture deformity of hand and consequent disability. There is not a satisfying method for management of the disease and standard invasive treatments such as collagenase injection and fasciectomy have high rates of failure and recurrence. Recently, focused extracorporeal shock wave therapy (ESWT) has been used successfully for reduction of pain and dysfunction in DC. We hypothesized that radial ESWT would benefit this condition and used it in a chronic case. The patient was a 64-year-old farmer with the history of DC in both hands for eight years. He experienced exacerbation in the flexion contracture of the fourth metacarpophalangeal (MCP) joint of his right hand in the past month. We treated the patient with six weekly sessions of radial shock wave. Extension restriction in the fourth MCP joint reached from $30^{\circ}$ to normal at the end of treatment and function of hand was also improved. Thus, radial ESWT should be considered and investigated for management of the DC in future studies. Keywords: Extracorporeal shock wave therapy, Dupuytren's contracture, Conservative treatments
\end{abstract}

Received: 16 May 2020, Accepted: 15 November 2020, ePublished: 30 June 2021

\section{Introduction}

Dupuytren's contracture (DC) or palmar fibromatosis is a benign progressive focal fibro-proliferative condition affecting palmar and digital aponeurosis of the hand (1). The condition begins with nodules which are more frequent in the base of ulnar fingers, mostly ring and long fingers, followed by formation of collagenous bands, and ultimately progressive contracture and deformity of the hand which could be definitely disabling, even interfering with activities of daily living (2). The exact mechanism of the diseases is unknown and interaction between genetic diathesis and environmental factors might determine the disease development (3).

Management of DC has been a challenging issue because most of the conservative treatments such as ultrasound, radiation, and oral chemotherapies are ineffective and invasive interventions such as injection of collagenase, percutaneous aponeurotomy, and open fasciotomy, as standard treatments for the disease, are all accompanied with high rates of recurrence $(4,5)$.

Extracorporeal shock wave therapy (ESWT) is a noninvasive modality which has been successfully used to treat musculoskeletal conditions such as plantar fasciitis and tendinopathies (6), as well as chronic wounds and burns with scar tissue $(7,8)$. Basic mechanism of the ESWT is transmission of short pulses of high pressure to the local tissues which has possibly a healing, angiogenesis, regenerative, and remodeling effect on the targeted tissues $(9,10)$. Currently, two modes of ESWT have been employed: focus and radial modes. Radial shockwave transmits much less energy, but is cheaper and more available than focus (9). The comparable efficacy of radial ESWT has made it popular in the field of musculoskeletal conditions (11). In this case, we report the effect of radial ESWT in a patient with DC.

\section{Case Presentation}

The patient was a 64-year-old male farmer referred to a physical medicine and rehabilitation clinic because of flexion contracture of the fourth metacarpophalangeal (MCP) joint of his right hand. He had noticed the symptoms of the disease since 8 years ago in both hands and recently experienced exacerbation in his right hand deformity. He did not note a positive family history for DC and did not have any of the known risk factors for DC, such as smoking, drinking, and diabetes. A 2-3 mm nodule and a thickened band was palpable at the base of the fourth MCP joint of the ring finger. Range of motion (ROM) of hand joints were measured using a goniometer. Fourth MCP had an extension restriction of 30 degrees

C 2021 The Author(s); Published by Shahrekord University of Medical Sciences. This is an open-access article distributed under the terms of the Creative Commons Attribution License (http://creativecommons.org/licenses/by/4.0), which permits unrestricted use, distribution, and reproduction in any medium, provided the original work is properly cited. 
(Figure 1). Other small joints of both hands showed normal ROM and his hands were not painful. Functional limitations were measured by the Persian version of quick DASH (Disabilities of the Arm, Shoulder and Hand) questionnaire before and one week after treatment completion.

Shock wave therapy was performed by the radial head of the device (Doulith SD1) for six weekly sessions. Each session consisted of 5000 shocks with the pressure of 5 bars and frequency of $15 \mathrm{~Hz}$. The shock waves were applied over nodule and band when tension was exerted over the contracture. ROM of fourth MCP reached to normal after six treatment sessions (Figure 1). Score of quick DASH questionnaire showed improvement of hand function one week after treatment (before treatment: 24 , one week after treatment: 18).

The DASH is a 30 -item questionnaire that assesses a patient>s ability to perform certain upper extremity activities. This questionnaire is a self-report tool by which patients can assess the difficulty and interference in daily life on a 5-point Likert scale.

\section{Discussion}

Recently, Knobloch et al proposed that focus shock waves can be used for management of DC (12), as this modality has beneficial effects on disorders with similar pathophysiology such as Peyronie's disease and Ledderhose's disease (plantar fibromatosis) $(13,14)$. Also, two studies evaluated the effect of focus ESWT on DC: a clinical trial which compared ESWT, high energy laser


Figure 1. Flexion contracture in a patient with Dupuytren's contracture before treatment with radial extracorporeal shock wave (A); Flexion contracture in a patient with Dupuytren's contracture one week after treatment with radial extracorporeal shock wave (B) therapy and stretching (15), and an observational study on the use of focus shock waves on palmar nodules of the disease (4). Both studies concluded that ESWT reduces pain and improves contractions and function at short term follow-ups.

As far as the researchers investigated, radial ESWT has not yet been used in DC. As it is a less expensive and more available system and has shown promising results in treatment of musculoskeletal diseases (11), we used this modality in a patient with DC for managing his contracture and disability.

We witnessed that radial ESWT fairly repaired the restricted ROM of MCP and somewhat improved the function of hand in a patient with DC. Although a robust conclusion has to be based on clinical trial studies, because of limited number of patients with DC, case reports and case series can be valuable sources of data to guide clinical practice and to found clinical studies. Radial ESWT is a non-invasive and inexpensive modality, which seems helpful to resolve contractions and improve hand function in patients with DC in short term. Also, well-designed clinical trials are recommended to compare long-term effects of radial and focused ESWT on DC.

\section{Conclusion}

Use of radial ESWT should be investigated for management of DC as a noninvasive, less expensive, and available modality to postpone invasive treatments.

Conflict of Interests

Authors declare that they have no conflict of interests.

\section{Ethical Approval}

All the experiments were conducted in accordance with the ethical guidelines on human experimentation. The Research Ethics Committee of Isfahan University of Medical Sciences, approved the study protocol (IR.MUI.MED.REC.1400.488).

\section{Authors' Contribution}

PT contributed to the design of the study, NS contributed to the data acquisition, and SGH drafted the manuscript. All authors critically revised the manuscript and approved its final version.

\section{Funding/Support}

This study was not funded by any organization.

References

1. Nunn AC, Schreuder FB. Dupuytren's contracture: emerging insight into a Viking disease. Hand Surg. 2014;19(3):48190. doi: 10.1142/s0218810414300058.

2. Mella JR, Guo L, Hung V. Dupuytren's contracture: an evidence based review. Ann Plast Surg. 2018;81(6S Suppl 1):S97-S101. doi: 10.1097/sap.0000000000001607.

3. Amadio CR, Magalhães BM, Yazawa F, Simone K. Dupuytren's disease: what dermatologists need to know. An Bras Dermatol. 2018;93(5):773-4. doi: 10.1590/abd18064841.20188035 .

4. Aykut S, Aydın C, Öztürk K, Arslanoğlu F, Kılınç CY. Extracorporeal shock wave therapy in Dupuytren's disease. Sisli Etfal Hastan Tip Bul. 2018;52(2):124-8. doi: 10.14744/ 
semb.2017.58076.

5. Mafi R, Hindocha S, Khan W. Recent surgical and medical advances in the treatment of Dupuytren's disease - a systematic review of the literature. Open Orthop J. 2012;6:77-82. doi: 10.2174/1874325001206010077.

6. Reilly JM, Bluman E, Tenforde AS. Effect of shockwave treatment for management of upper and lower extremity musculoskeletal conditions: a narrative review. PM R. 2018;10(12):1385-403. doi: 10.1016/j.pmrj.2018.05.007.

7. Fioramonti P, Cigna E, Onesti MG, Fino P, Fallico N, Scuderi N. Extracorporeal shock wave therapy for the management of burn scars. Dermatol Surg. 2012;38(5):77882. doi: $10.1111 / j .1524-4725.2012 .02355 . x$.

8. Zhang L, Weng C, Zhao Z, Fu X. Extracorporeal shock wave therapy for chronic wounds: a systematic review and metaanalysis of randomized controlled trials. Wound Repair Regen. 2017;25(4):697-706. doi: 10.1111/wrr.12566.

9. Lohrer H, Nauck T, Korakakis V, Malliaropoulos N. Historical ESWT paradigms are overcome: a narrative review. Biomed Res Int. 2016;2016:3850461. doi: 10.1155/2016/3850461

10. Visco V, Vulpiani MC, Torrisi MR, Ferretti A, Pavan A, Vetrano M. Experimental studies on the biological effects of extracorporeal shock wave therapy on tendon models. A review of the literature. Muscles Ligaments Tendons J.
2014;4(3):357-61.

11. Foldager CB, Kearney C, Spector M. Clinical application of extracorporeal shock wave therapy in orthopedics: focused versus unfocused shock waves. Ultrasound Med Biol. 2012;38(10):1673-80. doi: 10.1016/j. ultrasmedbio.2012.06.004.

12. Knobloch K, Kuehn M, Vogt PM. Focused extracorporeal shockwave therapy in Dupuytren's disease--a hypothesis. Med Hypotheses. 2011;76(5):635-7. doi: 10.1016/j. mehy.2011.01.018.

13. Knobloch K, Vogt PM. High-energy focussed extracorporeal shockwave therapy reduces pain in plantar fibromatosis (Ledderhose's disease). BMC Res Notes. 2012;5:542. doi: 10.1186/1756-0500-5-542.

14. Srirangam SJ, Manikandan R, Hussain J, Collins GN, O'Reilly PH. Long-term results of extracorporeal shockwave therapy for Peyronie's disease. J Endourol. 2006;20(11):8804. doi: 10.1089/end.2006.20.880.

15. Notarnicola A, Maccagnano G, Rifino F, Pesce V, Gallone MF, Covelli I, et al. Short-term effect of shockwave therapy, temperature controlled high energy adjustable multi-mode emission laser or stretching in Dupuytren's disease: a prospective randomized clinical trial. J Biol Regul Homeost Agents. 2017;31(3):775-84. 